ARTICLE

\title{
Ganoderic acid A is the effective ingredient of Ganoderma triterpenes in retarding renal cyst development in polycystic kidney disease
}

Jia Meng ${ }^{1}$, Sai-zhen Wang ${ }^{2,3}$, Jin-zhao He ${ }^{1}$, Shuai Zhu ${ }^{1}$, Bo-yue Huang ${ }^{1}$, Shu-yuan Wang ${ }^{1}$, Min Li ${ }^{1}$, Hong Zhou ${ }^{1}$, Shu-qian Lin ${ }^{2,3}$ and Bao-xue Yang ${ }^{1,4}$

\begin{abstract}
Autosomal dominant polycystic kidney disease (ADPKD) is one of the most common life-threatening monogenetic diseases characterized by progressive enlargement of fluid-filled renal cysts. Our previous study has shown that Ganoderma triterpenes (GT) retards PKD renal cyst development. In the present study we identified the effective ingredient of GT in suppression of kidney cyst development. Using an in vitro MDCK cystogenesis model, we identified ganoderic acid A (GA-A) as the most promising candidate among the 12 ganoderic acid (GA) monomers. We further showed that GA-A $(6.25-100 \mu \mathrm{M})$ significantly inhibited cyst growth in MDCK cyst model and embryonic kidney cyst model in vitro, and the inhibitory effect was reversible. In kidney-specific Pkd1 knockout (kPKD) mice displaying severe cystic kidney disease, administration of GA-A $\left(50 \mathrm{mg} \cdot \mathrm{kg}^{-1} \cdot \mathrm{d}^{-1}\right.$, sc) significantly attenuated renal cyst development. In both MDCK cells and kidney of kPKD mice, we revealed that GA-A dose-dependently downregulated the Ras/MAPK signaling pathway. The expression of proliferating cell nuclear antigen (PCNA) was also suppressed, suggesting a possible effect of GA-A on cell proliferation. These experimental data suggest that GA-A may be the main ingredient of GT as a potential therapeutic reagent for treating ADPKD.
\end{abstract}

Keywords: ADPKD; Ganoderma triterpenes; Ganoderic acids; Ganoderic acid A; MDCK; kPKD mice; Ras/MAPK signaling pathway; 8Br-cAMP

Acta Pharmacologica Sinica (2020) 41:782-790; https://doi.org/10.1038/s41401-019-0329-2

\section{INTRODUCTION}

Autosomal dominant polycystic kidney disease (ADPKD) is one of the most common genetic diseases and occurs in 1:1000 to 1:400 individuals, affecting estimated 12.5 million people worldwide $[1,2]$. ADPKD is caused by mutations in Pkd1 ( 85\% of cases) or Pkd2 ( 15\% of cases), which encode polycystin-1 or polycystin-2, respectively $[3,4]$. ADPKD is characterized by the continuous development of renal fluid-filled cysts derived from tubular epithelial cells [5]. The growth of cysts results in the severe destruction of the renal parenchyma and ultimately leads to the loss of renal function and kidney failure [6]. Patients with ADPKD may develop end-stage renal disease (ESRD) by 60 years of age. ADPKD is considered as the fourth most common renal disease requiring renal replacement therapy $[7,8]$ due to the very limited pharmacological approaches that are effective in preventing or reversing PKD progression [9]. Although tolvaptan was approved by the Food and Drug Administration (FDA) in 2018 for treating ADPKD, the potential side effects are still of concern [10]. Thus, advances in research are still required to discover promising therapeutic agents for ADPKD [11].

Our previous study found that Ganoderma triterpenes (GT), the major secondary metabolites of Ganoderma lucidum (G. lucidum), have pharmacological activity that inhibit renal cyst development in PKD mainly by promoting cyst epithelial cell differentiation and downregulating intracellular cyclic adenosine monophosphate (cAMP) levels and the Ras/mitogen-activated protein kinase (Ras/ MAPK) pathway [12]. However, owing to the diverse components and complex structures of GT, which consist of hundreds of single compounds, it is still unclear what compounds in GT are mainly responsible for anti-cyst pharmacological activity [13]. The pharmacological study of the purified triterpene monomer will determine the key component of GT and increase the understanding of its critical anti-cyst effect, which will benefit the development of new drugs for treating ADPKD.

Several studies have shown that Ganoderic acids (GA) are the major components of GT [14] and exhibit extraordinary pharmacological activities, especially anticancer activity, that affect multiple signaling pathways involved in the pathogenesis of ADPKD [15-18]. Hence, in this study, 12 monomers were isolated from GA, and their anti-cyst activity was assessed using in vitro and in vivo cyst models [19]. The experimental results showed that GA-A had optimal inhibitory activity against cyst development, suggesting that GA-A might be the active GT ingredient. It was also confirmed that GA-A was able to attenuate renal cyst

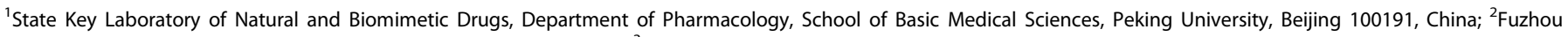

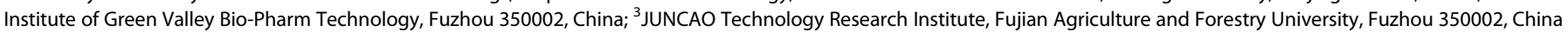
and ${ }^{4}$ Key Laboratory of Molecular Cardiovascular Sciences, Ministry of Education, Beijing 100191, China

Correspondence: Bao-xue Yang (baoxue@bjmu.edu.cn)

Received: 25 August 2019 Accepted: 31 October 2019

Published online: 7 January 2020 
development by downregulating the Ras/MAPK signaling pathway and cell proliferation. The data in this study suggest that GA-A might be a promising candidate drug for treating ADPKD.

\section{MATERIALS AND METHODS}

Chemicals and reagents

All 12 GA monomers listed in Table 1 were kindly provided by the JUNCAO Technology Research Institute of Fujian Agriculture and
Forestry University. The purity of the GA monomers was determined by HPLC to be greater than $98 \%$.

Forskolin (FSK) and 8-bromoadenosine $3^{\prime}, 5^{\prime}$-cyclic monophosphate (8-Br-cAMP) were obtained from Sigma-Aldrich (St. Louis, MO, USA) and dissolved in dimethyl sulfoxide (DMSO; SigmaAldrich) to prepare a $100 \mathrm{mM}$ stock solution, which was stored in a freezer at $-20^{\circ} \mathrm{C}$. The following antibodies were purchased from Santa Cruz Biotechnology (Santa Cruz, CA, USA): anti-B-raf (sc-166), anti-Raf-1 (sc-227), anti-p-ERK (sc-7383), anti-ERK2 (sc-154),

Table 1. Information of 12 puried GA monomers.

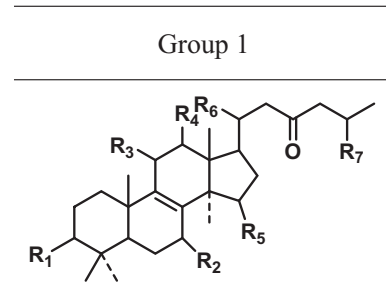

$\begin{array}{lllllllll}\text { Compound } & \mathrm{R} 1 & \mathrm{R} 2 & \mathrm{R} 3 & \mathrm{R} 4 & \mathrm{R} 5 & \mathrm{R} 6 & \mathrm{R} 7\end{array}$

R7

Inhibition ratio (\%)

\begin{tabular}{lcccccccc} 
Ganoderic acid A & $=\mathrm{O}$ & $\beta-\mathrm{OH}$ & $=\mathrm{O}$ & $\mathrm{H}$ & $\alpha-\mathrm{OH}$ & $\beta-\mathrm{CH}_{3}$ & $\mathrm{COOH}$ & 46.66 \\
\hline Ganoderic acid B & $\beta-\mathrm{OH}$ & $\beta-\mathrm{OH}$ & $=\mathrm{O}$ & $\mathrm{H}$ & $=\mathrm{O}$ & $\beta-\mathrm{CH}_{3}$ & $\mathrm{COOH}$ & 3.82 \\
\hline Ganoderic acid C2 & $\beta-\mathrm{OH}$ & $\beta-\mathrm{OH}$ & $=\mathrm{O}$ & $\mathrm{H}$ & $\alpha-\mathrm{OH}$ & $\alpha-\mathrm{CH}_{3}$ & $\mathrm{COOH}$ & 13.94 \\
\hline
\end{tabular}

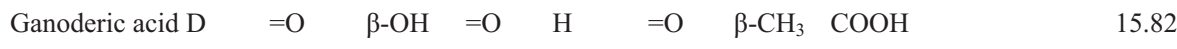

$\begin{array}{lllllll}\text { Ganoderic acid } \mathrm{F} \quad \mathrm{O} & =\mathrm{O} & \mathrm{H} & =\mathrm{O} & \beta-\mathrm{CH}_{3} & =\mathrm{O} & \mathrm{COOH}\end{array}$

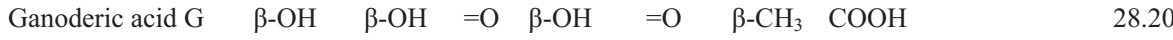

\begin{tabular}{|c|c|c|c|c|c|c|c|c|c|}
\hline Group 2 & Compound & $\mathrm{R} 1$ & $\mathrm{R} 2$ & R3 & R4 & R5 & R6 & R7 & Inhibition ratio $(\%)$ \\
\hline & Ganoderenic acid A & $=\mathrm{O}$ & $\beta-\mathrm{OH}$ & $\mathrm{H}$ & $\alpha-\mathrm{OH}$ & $\beta-\mathrm{CH}_{3}$ & $=\mathrm{O}$ & $\mathrm{COOH}$ & -2.57 \\
\hline & Ganoderenic acid B & $\beta-\mathrm{OH}$ & $\beta-\mathrm{OH}$ & $\mathrm{H}$ & $=\mathrm{O}$ & $\beta-\mathrm{CH}_{3}$ & $=\mathrm{O}$ & $\mathrm{COOH}$ & -1.31 \\
\hline & Ganoderenic acid D & $=\mathrm{O}$ & $\beta-\mathrm{OH}$ & $\mathrm{H}$ & $=\mathrm{O}$ & $\beta-\mathrm{CH}_{3}$ & $=\mathrm{O}$ & $\mathrm{COOH}$ & 4.45 \\
\hline & Ganoderenic acid F & $=\mathrm{O}$ & $=\mathrm{O}$ & $\mathrm{H}$ & $=\mathrm{O}$ & $\beta-\mathrm{CH}_{3}$ & $=\mathrm{O}$ & $\mathrm{COOH}$ & -1.67 \\
\hline
\end{tabular}

\begin{tabular}{l|cccccccccc}
\hline Group3 & Compound & $\mathrm{R} 1$ & $\mathrm{R} 2$ & $\mathrm{R} 3$ & $\mathrm{R} 4$ & $\mathrm{R} 5$ & $\mathrm{R} 6$ & $\mathrm{R} 7$ & $\mathrm{R} 8$ & Inhibition ratio (\%) \\
\hline & Ganoderic acid DM & $=\mathrm{O}$ & $=\mathrm{O}$ & $\mathrm{H}$ & $\mathrm{H}$ & $\mathrm{H}$ & $\alpha-\mathrm{OH}$ & $\mathrm{H}$ & $\mathrm{COOH}$ & 30.88
\end{tabular}<smiles>[R]C([R])CC(C)C(C)C(C)(C)C([R])CC</smiles>

\begin{tabular}{c|cccccccccc}
\hline Group4 & Compound & $\mathrm{R} 1$ & $\mathrm{R} 2$ & $\mathrm{R} 3$ & $\mathrm{R} 4$ & $\mathrm{R} 5$ & $\mathrm{R} 6$ & $\mathrm{R} 7$ & $\mathrm{R} 8$ & Inhibition ratio (\%) \\
\hline & Ganoderic acid T & $\alpha-\mathrm{O}-\mathrm{Ac}$ & $\alpha-\mathrm{O}-\mathrm{Ac}$ & $\mathrm{H}$ & $\alpha-\mathrm{CH}_{3}$ & $\beta-\mathrm{O}-\mathrm{Ac}$ & $\mathrm{H}$ & $\mathrm{COOH}$ & $\mathrm{CH}_{3}$ & 32.89
\end{tabular}


anti-Egr-1 (sc-110), and anti-c-fos (sc-253). The antibody against GAPDH (AC033) was purchased from ABclonal (Wuhan, China), and the antibody against proliferating cell nuclear antigen (PCNA) (D3H8P) was purchased from Cell Signaling Technology (Danvers, $M A)$. The goat anti-mouse IgG was purchased from Santa Cruz Biotechnology (Santa Cruz, CA, USA). The goat anti-rabbit IgG was purchased from Cell Signaling Technology (Danvers, MA, USA).

\section{Cell culture}

Type I Madin-Darby canine kidney (MDCK) cells were purchased from the American Type Culture Collection (ATCC, VA, USA). The type I MDCK cells were cultured in Dulbecco's modified Eagle's medium (DMEM) containing 10\% fetal bovine serum (FBS), $2 \mathrm{mM}$ glutamine, and $1 \%$ penicillin-streptomycin at $37{ }^{\circ} \mathrm{C}$ in a humidified cell culture incubator with $5 \% \mathrm{CO}_{2}$ and $95 \%$ air. The cells were seeded at a density of $2 \times 10^{4}$ cells $/ \mathrm{mL}$ in a $60-\mathrm{mm}$ plate (Corning, NY, USA), cultured until they reached $70 \%$ to $80 \%$ confluence, and prepared for use.

Cell proliferation analysis

A CCK-8 assay kit (Dojindo, Tokyo, Japan) was used to evaluate the cell proliferation of MDCK cells. Cells were plated in 96-well plates at a density of $1 \times 10^{4}$ cells/well. At each time point, $100 \mu \mathrm{L} \mathrm{CCK-8}$ solution diluted $1 / 10$ with DMEM containing $10 \%$ FBS was added to each well, and the cells were incubated for $2 \mathrm{~h}$ at $37^{\circ} \mathrm{C}$. The absorbance at $450 \mathrm{~nm}$ was measured with a microplate reader (MQX200; BioTek Instruments, Winooski, VT, USA).

\section{MDCK cyst model}

Approximately 400 MDCK cells were suspended in $0.4 \mathrm{~mL}$ of ice-cold MEM (Sigma-Aldrich, St. Louis, MO, USA) containing $2.9 \mathrm{mg} / \mathrm{mL}$ collagen (PureCol; Inamed Biomaterials, Fremont, CA, USA), $10 \mathrm{mM}$ HEPES, $100 \mathrm{U} / \mathrm{mL}$ penicillin, and $100 \mu \mathrm{g} / \mathrm{mL}$ streptomycin ( $\mathrm{pH} 7.4$ ) in each well of a 24-well plate (Corning, NY, USA). Dulbecco's modified Eagle's medium-F12 medium (DMEM-F12) containing $10 \%$ FBS and $10 \mu \mathrm{M}$ FSK without or with GA monomers was added to each well and exchanged every $12 \mathrm{~h}$. The relevant procedures were performed as previously described [20]. Micrographs of the cysts ( $\geq 30 /$ group) were obtained with an inverted microscope (Nikon TE2000-S; Nikon Instruments, Melville, NY) on days $4,6,8,10$, and 12 . The diameters of the cysts were measured and analyzed using Photoshop CS5 software (Photoshop CS5, Adobe Systems Inc. CA, USA).

\section{Embryonic kidney cyst model}

Wild-type ICR mouse kidneys from embryonic day 13.5 were dissected and placed on Transwell filters (Corning, NY, USA) as previously described $[21,22]$. The lower chambers were filled with DMEM-F12 medium supplemented with $2 \mathrm{mM}$ L-glutamine, $10 \mathrm{mM}$ HEPES, $5 \mu \mathrm{g} / \mathrm{mL}$ insulin, $5 \mu \mathrm{g} / \mathrm{mL}$ transferrin, $2.8 \mathrm{nM}$ selenium, $25 \mathrm{ng} / \mathrm{mL}$ prostaglandin $\mathrm{E}, 32 \mathrm{pg} / \mathrm{mL} \mathrm{T} 3,250 \mathrm{U} / \mathrm{mL}$ penicillin, $250 \mu \mathrm{g} / \mathrm{mL}$ streptomycin, and $100 \mu \mathrm{M}$ 8'-Br-cAMP. The medium was replaced every $12 \mathrm{~h}$. The kidneys were photographed using a Nikon inverted microscope (Nikon TE2000-S; Nikon Instruments, Melville, NY) every 2 days. The cyst area and total kidney area were measured using Image-Pro Plus 6.0 software (Media Cybernetics, Rockville, MD, USA). All animal experiments were conducted according to the animal protocols approved by the Peking University Health Science Center Committee on Animal Research.

\section{ADPKD mouse model}

$P k d 1^{f l o x}$ mice (from the Yale PKD Center) and Ksp-Cre transgenic mice (from UT Southwestern O'Brien Center) with a C57BL/6 genetic background were generated as previously described $[12,23]$. The kidney-specific Cre recombinase was expressed under the control of the Ksp-cadherin promoter. Wild-type $\left(P k d 1^{+/+} ; \mathrm{Ksp}\right.$ $\mathrm{Cre})$ mice and KPKD ( $\mathrm{Pkd} 1^{\text {flox/flox }}$; Ksp-Cre) mice were generated by self-crossing of $P k d 1^{f l o x /+} ; K s p$-Cre mice. Neonatal mice (age 1 day) were genotyped by genomic polymerase chain reaction. The wildtype and KPKD mice were each divided into 2 groups: a saline DMSO vehicle control group ( $0.02 \mathrm{~mL} /$ injection) and a GA-A group (50 mg. $\mathrm{kg}^{-1} \cdot \mathrm{d}^{-1}$ ) ( $\geq 5$ mice/group). The chemicals were administered by a subcutaneous injection into the backs of the mice every $24 \mathrm{~h}$ using a $1-\mathrm{mL}$ insulin syringe. At postnatal day 4 , the mice were weighed and sacrificed. Tissues were harvested and weighed.

Cell lysate preparation and Western blotting

The specific procedures used for the cell and tissue lysate preparation and Western blotting analysis were performed as previously described [24]. Blots were incubated with antibodies against B-raf, raf-1, p-ERK, ERK2, Egr-1, c-fos, PCNA and GAPDH. The relative protein expression was quantified according to the optical density using ImageJ software (NIH, MD, USA). All experiments were repeated at least six times.

\section{Statistical analysis}

All results are presented as the mean \pm SEM. Each experiment was performed at least 3 times. For multiple comparisons, statistical analysis was performed by using Student's $t$-test or two-way ANOVA. $P$-values $<0.05$ were considered statistically significant. The statistical analyses were plotted with GraphPad Prism software (GraphPad Software, Inc., San Diego, CA, USA).

\section{RESULTS}

GA-A was identified as the active GT monomer using the MDCK cyst model

The anti-cyst effects of GA monomers were evaluated using the MDCK cyst model. MDCK cells were cultivated in threedimensional (3D) collagen I gels in the presence of $10 \mu \mathrm{M}$ FSK for 5 days, during which fluid-filled cysts developed that were spontaneously surrounded by monolayer cells. Then, the cysts were incubated with $10 \mu \mathrm{M}$ FSK and simultaneously treated with $10 \mu \mathrm{M}$ GA monomers from day 5 to day 12. During FSK stimulation, the cysts progressively expanded to 400 to $500 \mu \mathrm{m}$ in diameter by day 12. MDCK cyst enlargement was inhibited by Ganoderic acid A (GA-A), Ganoderic acid G (GA-G), Ganoderic acid D (GA-D), Ganoderic acid C2 (GA-C2), Ganoderic acid T (GA-T) and Ganoderic acid DM (GA-DM). The specific activity was assessed according to the cyst inhibition ratio on day 12 , as shown in Table 1. GA-A showed the greatest inhibitory effect (Fig. 1a, b). The cytotoxicity of the GAs at $6.25,12.5,25,50$, and $100 \mu \mathrm{M}$ was evaluated in MDCK cells using a CCK-8 assay. After $24 \mathrm{~h}$ of treatment, the 12 GA monomers did not affect MDCK cell viability at concentrations below $100 \mu \mathrm{M}$ (Fig. 1c). Therefore, GA-A was selected for further study.

GA-A dose-dependently inhibited cyst formation and enlargement in the MDCK cyst model

To determine the inhibitory activity of GA-A on cyst formation, MDCK cells were cultured in 3D collagen I gels and were treated either with or without $25 \mu \mathrm{M}$ GA-A in the presence of $10 \mu \mathrm{M}$ FSK over a 6-day time span. On day 6, the number of cysts (diameter $>50 \mu \mathrm{m})$ and non-cyst colonies were counted. There were fewer cysts in the GA-A-treated group than in the control group (Fig. 2a). There was no difference in the total number of colonies (including colonies of cells and cysts) between the 2 groups, supporting the above results that showed that GA-A was not toxic to MDCK cells.

To confirm its inhibitory effect on cyst growth, GA-A at different concentrations $(6.25,25$, and $100 \mu \mathrm{M})$ was added at day 5 into the FSK-treated MDCK cyst culture. The cysts were photographed and measured every two days. MDCK cyst enlargement was significantly inhibited by GA-A in a dose-dependent manner (Fig. 2b, $c$ ). After removing GA-A by washing the cysts on day 8 , the MDCK 
a

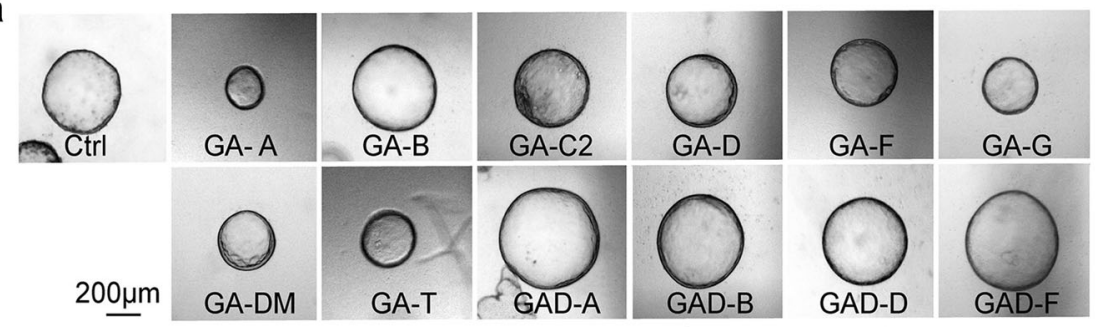

Representative graphs of cysts on Day12
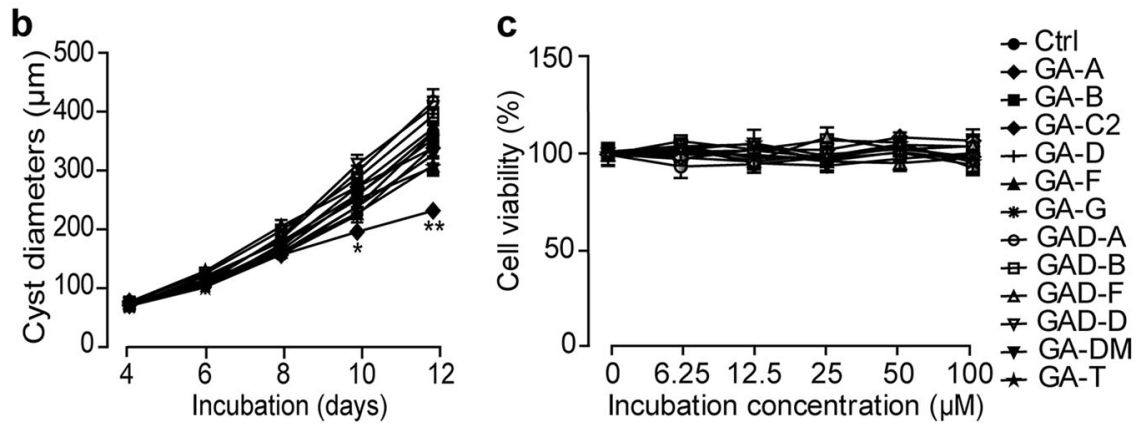

Fig. 1 GA-A was identified as the active GT monomer using the MDCK cyst model. a Representative light micrographs of MDCK cysts in collagen gel on day 12 treated with the 12 GA monomers for the initial screening. GA-A, GA-B, GA-C2, GA-D, GA-F, GA-G, GA-DM, and GA-T are the abbreviations for Ganoderic acid A, Ganoderic acid B, Ganoderic acid C2, Ganoderic acid D, Ganoderic acid F, Ganoderic acid G, Ganoderic acid DM and Ganoderic acid T, respectively. GAD-A, GAD-B, GAD-D, and GAD-F are the abbreviations for Ganoderenic acid A, Ganoderenic acid $B$, Ganoderenic acid D and Ganoderenic acid F, respectively. Scale bars, $200 \mu \mathrm{m}$. b Cyst diameters after exposure to different GA monomers $(10 \mu \mathrm{M})$ for 8 days from day 5 to day 12 . Data are presented as the mean \pm SEM (analysis of 30 cysts/time point). ${ }^{*} P<0.05,{ }^{* *} P<0.01$ vs Ctrl (only GA-A statistical results are shown). c Cell viability of MDCK cells treated with $6.25,12.5,25$, or $100 \mu \mathrm{M}$ GA monomer for $24 \mathrm{~h}$. Data are presented as the mean \pm SEM. $n=5$.

a

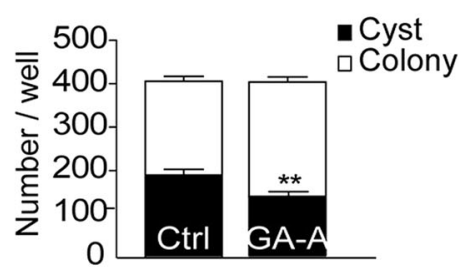

C
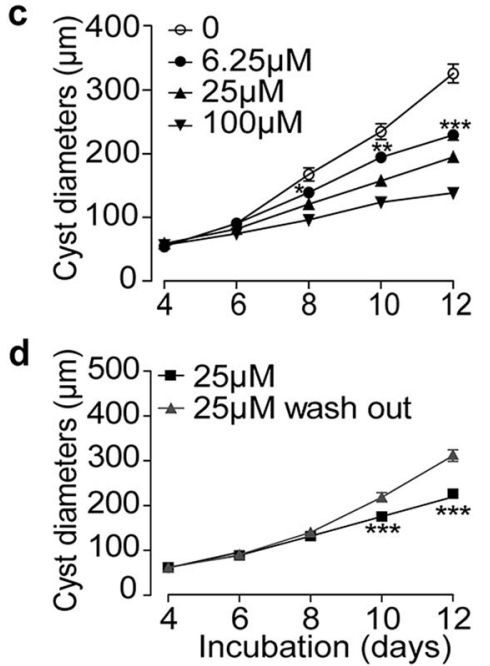

b
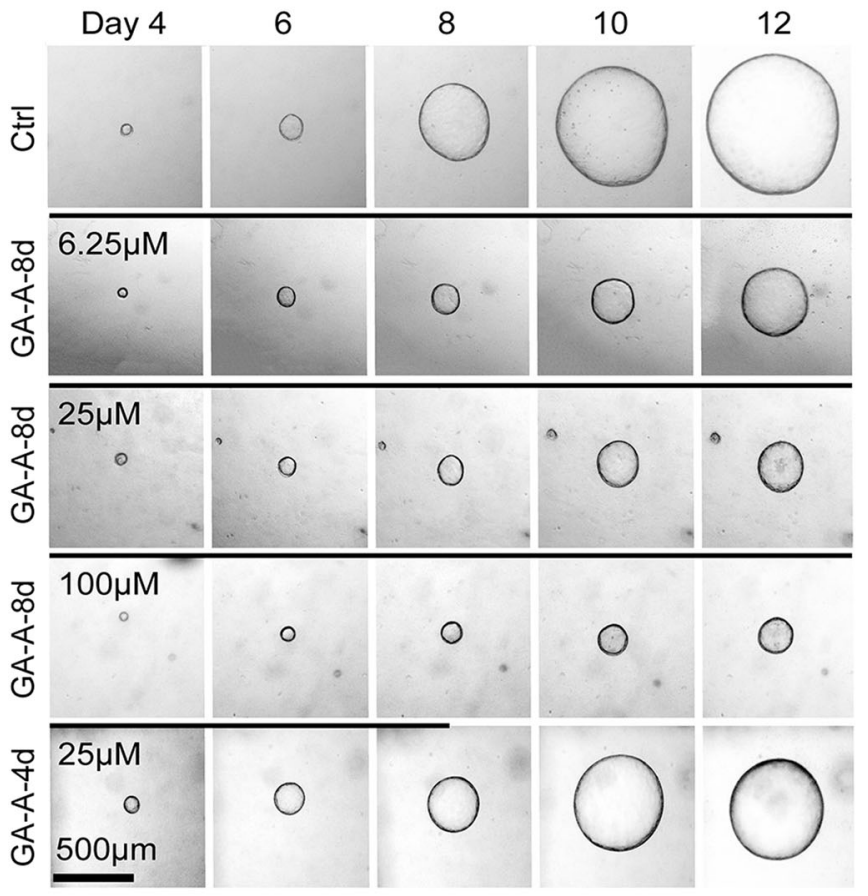

Fig. 2 GA-A dose-dependently inhibited cyst formation and enlargement in the MDCK cyst model. a The number of MDCK cysts and the total numbers of colonies per well untreated (Ctrl) or treated with GA-A $(25 \mu \mathrm{M})$. Data are presented as the mean \pm SEM. ${ }^{* *} P<0.01$ vs $C$ trl. $n=$ 3. b Representative light micrographs of MDCK cysts in collagen gel from day 4 to day 12 that were untreated or treated with different GA-A concentrations $(6.25,25$, and $100 \mu \mathrm{M})$. The thick black lines indicate the culture time with GA-A. Scale bar, $500 \mu \mathrm{m}$. c Diameters of cysts without (Ctrl) or with exposure to different GA-A concentrations. Data are presented as the mean \pm SEM (analysis of 30 cysts/time point). ${ }^{*} P<0.05$, ${ }^{* *} P<0.01,{ }^{* * *} P<0.001$ vs Ctrl. d Cyst inhibition was reversible after GA-A was removed on day 8 (GA-A-4 d). Data are presented as the mean \pm SEM (analysis of 30 cysts/time point). ${ }^{* *} P<0.001$ vs the continuously GA-A-treated group. 
a

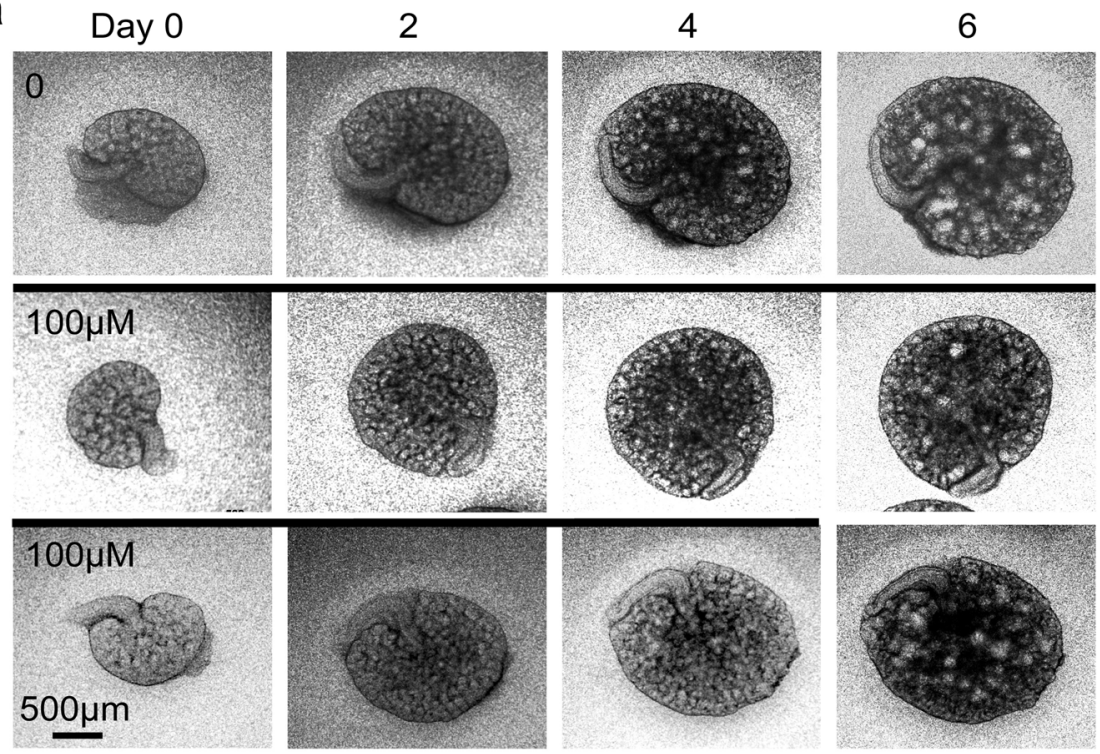

b

GA-A $(\mu \mathrm{M})$

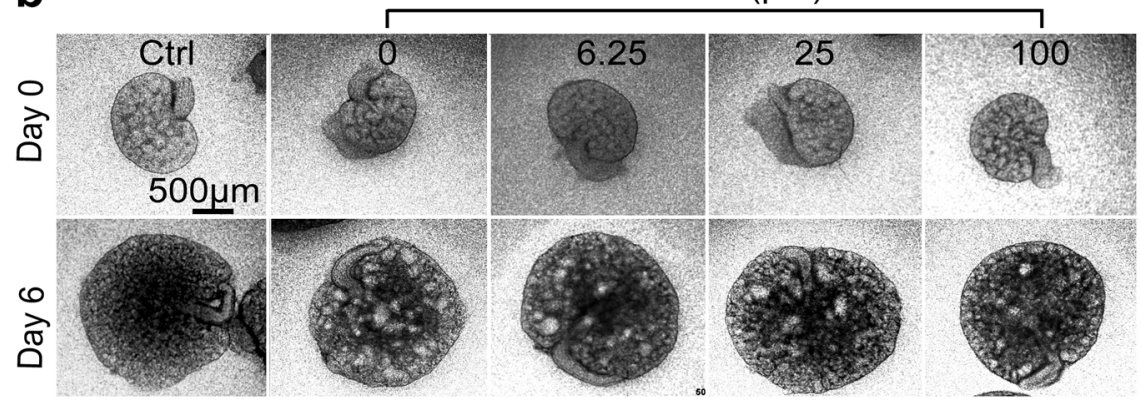

C

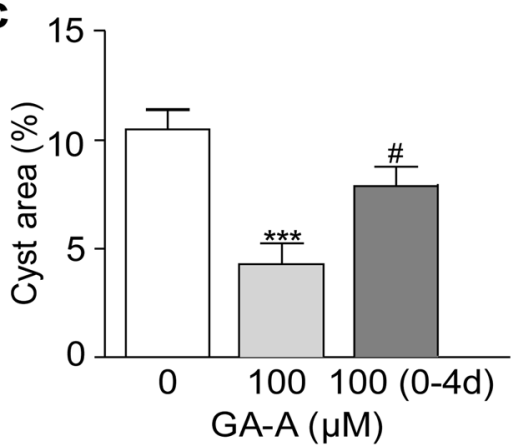

d

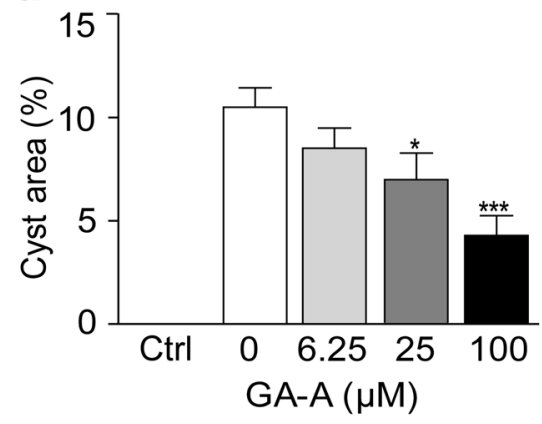

Fig. 3 GA-A inhibited renal cyst development in an embryonic kidney cyst model. a Embryonic kidneys cultured with $100 \mu \mathrm{M} 8$-Br-cAMP as $\mathrm{Ctrl}$ (top) or treated with $100 \mu \mathrm{M}$ GA-A from day 0 to 6 (middle) or from day 0 to 4 (bottom). The thick black lines indicate the culture time with GA-A. Scale bar, $500 \mu \mathrm{m}$. b Representative light micrographs of embryonic kidneys cultivated as the negative Ctrl (left) without $100 \mu \mathrm{M} 8$-BrcAMP or exposed to different GA-A concentrations $(0,6.25,25$, and $100 \mu \mathrm{M})$ in the presence of $100 \mu \mathrm{M} 8$-Br-cAMP at day 0 and day 6. Scale bar, $500 \mu \mathrm{m}$. c Fractional cyst area (\%) of kidneys treated without (Ctrl) or with $100 \mu \mathrm{M}$ GA-A from day 0 to day 6 or from day 0 to day 4 . Data are presented as the mean \pm SEM $(n=9)$. ${ }^{* * *} P<0.001$ vs. Ctrl. ${ }^{\#} P<0.05$ vs the group treated with GA-A from day $0-6$. d Fractional cyst area (\%) of kidneys treated as a negative Ctrl and with different GA-A concentrations from day 0 to 6 . Data are presented as the mean \pm SEM $(n=9)$. ${ }^{*} P<$ $0.05,{ }^{* * *} P<0.001$ vs GA-A $(0 \mu \mathrm{M})$.

cysts grew quickly again (Fig. 2d), suggesting that the inhibitory effect of GA-A on MDCK cyst enlargement was reversible.

GA-A inhibited renal cyst development in an embryonic kidney cyst model

The inhibitory effects of GA-A on renal cysts was also tested in an embryonic kidney model. Kidneys removed from wild-type ICR mice on embryonic day 13.5 were cultured on Transwell filters as previously described $[21,22]$. In the presence of 8 -Br-cAMP, numerous cystic structures rapidly developed in the cultured embryonic kidneys (Fig. 3a, top panel), while renal cyst development was significantly inhibited by $100 \mu \mathrm{M}$ GA-A (Fig. 3a, middle panel). After 6 days of continuous photo tracking, we quantified the fractional cyst area and found that GA-A did not affect kidney growth but remarkably reduced the number and sizes of renal cysts in a dose-dependent manner (Fig. 3b, d). After removing GA-A from the embryonic kidneys on day 4, cyst growth recommenced in the 8 -Br-cAMP-treated kidneys, demonstrating 
a

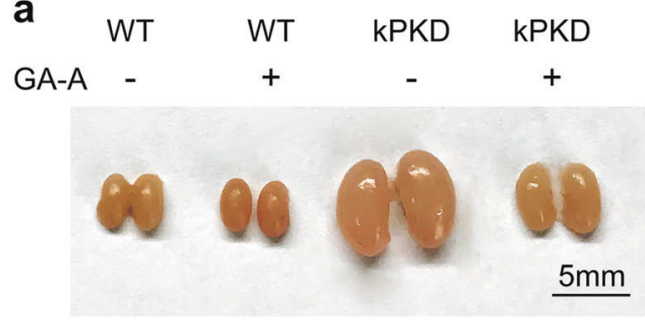

C

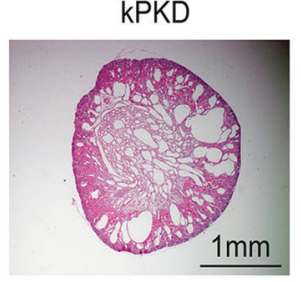

kPKD+GA-A

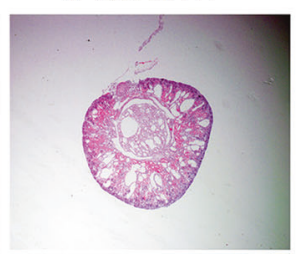

b

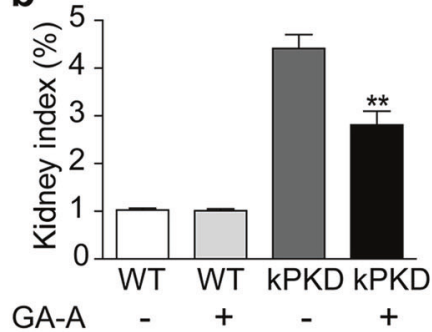

d

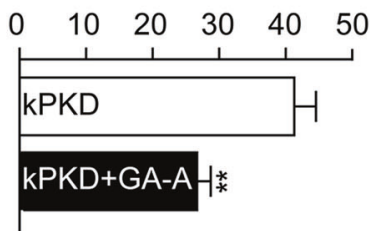

Fig. 4 GA-A repressed renal cyst development in an ADPKD mouse model. a Mouse kidneys from wild-type (WT) mice and kPKD mice at postnatal day 4 were treated with vehicle or $50 \mathrm{mg} \cdot \mathrm{kg}^{-1} \cdot \mathrm{d}^{-1} \mathrm{GA}-\mathrm{A}$ for 4 days. Scale bar, $5 \mathrm{~mm}$. b Kidney index (total kidney weight/body weight). Data are presented as the mean \pm SEM. $n=6$. ${ }^{*} P<0.01$ vs $\mathrm{kPKD}$ mice treated with vehicle. c Hematoxylin and eosin staining of kidneys in vehicle- or GA-A-treated kPKD mice. Scale bar, $1 \mathrm{~mm}$. d Fractional cyst area (cyst area/kidney area) in the same mice in c. Data are presented as the mean \pm SEM. $n \geq 6$. ${ }^{* *} P<0.01$ vs $\mathrm{kPKD}$ mice treated with vehicle.

that GA-A slowed cyst growth reversibly without affecting the viability of the tissue (Fig. 3a, bottom panel, and Fig. 3c).

GA-A repressed renal cyst development in an ADPKD mouse model

In the following experiments, kidney-specific Pkd1 knockout mice $\left(P k d 1^{\text {flox/flox }}\right.$ : Ksp-Cre; kPKD) were used to further investigate the inhibitory effects of GA-A on renal cysts in vivo. In kPKD mice, renal cysts began to appear on postnatal day 1 and resulted in progressive renal cyst disease [23]. Wild-type mice $\left(P k d 1^{+/+} ; \mathrm{Ksp}\right.$ Cre) and kPKD mice were treated with or without GA-A. GA-A was subcutaneously injected every $24 \mathrm{~h}$ at $50 \mathrm{mg} / \mathrm{kg}$ per day from postnatal day 1 until day 4 . During the 4 days of treatment, there were no significant differences in body weight or liver weight among the groups (data not shown). GA-A did not affect the kidney size in wild-type mice but decreased the kidney size in kPKD mice (Fig. 4a). GA-A significantly reduced the kidney index (total kidney weight/body weight) in kPKD mice (Fig. 4b). Hematoxylin and eosin staining of sections of kidneys showed that the kidneys were remarkably enlarged and contained numerous cysts in the KPKD mice. GA-A treatment reduced the sizes of the cysts and kidney masses noticeably in KPKD mice (Fig. 4c). The fractional cyst area in the kidneys of kPKD mice was decreased by approximately $40 \%$ by GA-A treatment (Fig. $4 d$ ).

GA-A downregulated FSK-induced activation of the Ras/MAPK signaling pathway in MDCK cells

The effect of GA-A on the Ras/MAPK pathway was evaluated by Western blotting. After serum starvation, the levels of the B-raf, pERK, Egr-1, and c-fos signaling molecules were significantly elevated, while Raf-1 expression was remarkably decreased in MDCK cells treated with $10 \mu \mathrm{M}$ FSK for $30 \mathrm{~min}$. GA-A treatment reversed these changes in a dose-dependent manner (Fig. 5a, b). These results indicated that GA-A represents the main active component of GT, which inhibits cyst development.

GA-A downregulated the Ras/MAPK signaling pathway and repressed cell proliferation in the kidneys in the ADPKD mouse model

To confirm the effect of GA-A on the Ras/MAPK signaling pathway in vivo, we detected key molecules, including p-ERK, ERK2, the upstream effector B-raf, and the downstream effector c-fos, in kidney tissue from the KPKD mouse model by Western blotting. The results (Fig. $5 c$, d) showed that GA-A treatment significantly downregulated the Ras/MAPK signaling pathway by reducing the expression of B-raf, p-ERK, and c-fos, which are usually excessively activated in ADPKD kidneys. GA-A had no detectable effect on the Ras/MAPK pathway in normal kidneys.

Cell proliferation is considered a major factor leading to cyst development in ADPKD as well. Therefore, we evaluated the effect of GA-A on the expression of PCNA, which is a marker of cell proliferation. The results showed that the PCNA level was significantly decreased by GA-A in the kidneys of KPKD mice but did not affect normal kidneys, indicating that the suppression of cellular proliferation may be involved in the effect of GA-A on ADPKD (Fig. 5c, d).

\section{DISCUSSION}

The aim of this study was to determine the most effective triterpene ingredient with anti-cyst activity among the available GA monomers. To identify the active GA monomers with anti-cyst activity in ADPKD, this study used the MDCK cyst model to screen the GA monomers in GT. The candidate monomers are listed in Table 1. All candidates share the same prototypical lanostane triterpenoid skeleton but have different side chains. The 12 candidates can be further specifically classified into four structural categories that represent the main structural compositions of GAs [25].

The monomers in group 1 contain double bonds between C-8 and $C-9$, a keto group at $C-23$, and substituent groups at $C-3,7,11$, $12,15,25$ corresponding to GA-A, GA-B, GA-C2, GA-D, GA-F and GA-G [26-29]. Of the six compounds in this group, GA-A exhibited the most significant inhibitory effect on MDCK cyst development at day 8 , and the inhibitory effect was significantly different at day 10. GA-B did not have an effect, while GA-C2, GA-D and GA-F showed a mild inhibitory effect, which was only revealed on day 12. The effect of GA-G was slightly less than that of GA-A and greater than that of the other four compounds. Except for GA-F, the R2 substituent at C-7 and the R3 substituent at C-11 in this group of compounds were beta hydroxyl and ketone, respectively, but the anti-cyst activity varied greatly. The inhibition ratio of GA-B 
a

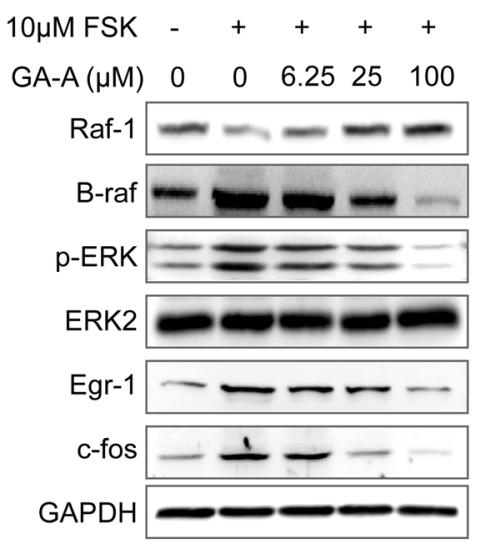

C

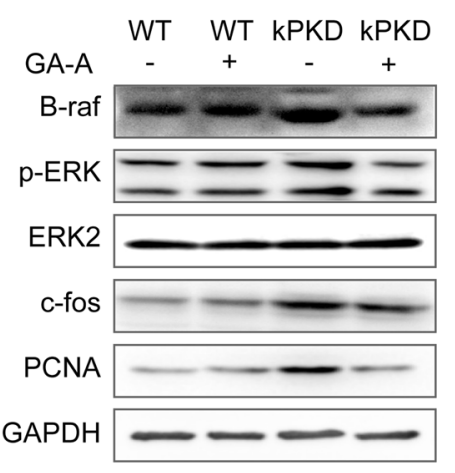

b

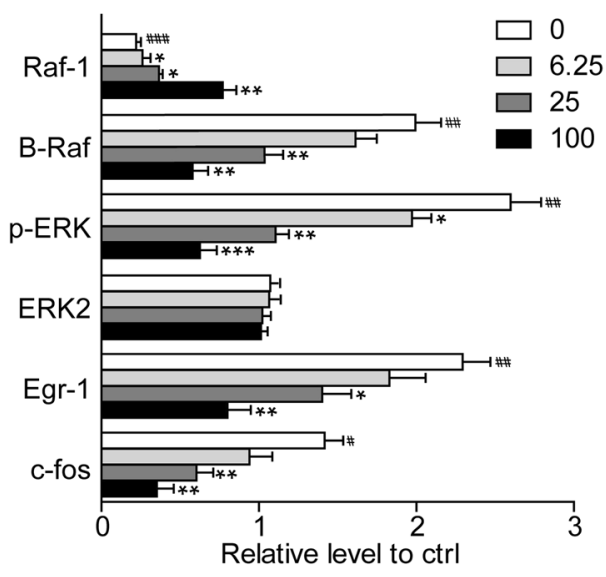

d

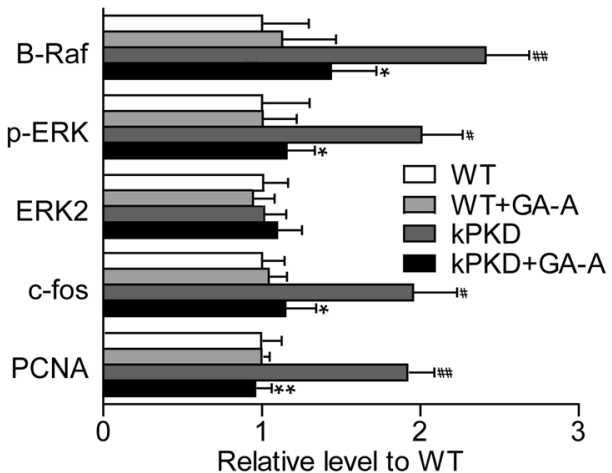

Fig. 5 GA-A downregulated the Ras/MAPK signaling pathway in the FSK-treated MDCK cells and kidneys in the ADPKD mouse model. a Representative Western blotting of signaling proteins in MDCK cells treated without (Ctrl) or with $10 \mu \mathrm{M}$ FSK; some cells were also treated with GA-A at the indicated concentrations $(6.25,25$, and $100 \mu \mathrm{M})$ for $30 \mathrm{~min}$. b Quantification of signaling protein expression shown in a. Data are presented as the mean \pm SEM. $n=6$. ${ }^{\#} P<0.05,{ }^{\# \#} P<0.01$ vs Ctrl. ${ }^{*} P<0.05,{ }^{* *} P<0.01$ vs the FSK-treated group only. c Representative Western blotting of signaling proteins in mouse kidneys without or with GA-A treatment. d Quantification of signaling protein expression shown in $c$. Data are presented as the mean \pm SEM. $n=6$. ${ }^{\#} P<0.05,{ }^{\# \#} P<0.01$ vs the WT group. ${ }^{*} P<0.05$, ${ }^{* *} P<0.01$ vs the kPKD group.

was as low as $3.82 \%$, but that of GA-A was $46.66 \%$. Furthermore, the inhibition ratio of GA-F was $21.19 \%$, and GA-F exhibited the second best anti-cyst activity in this group.

The structure and activity analysis indicated that the substituents of C-7 and C-11 had little effect on GA activity. When comparing the structure of GA-A with that of the other five compounds, we found that the specific substituents of C-3 and C15 seemed to make a difference. Compared with that of GA-A, the structure of GA-C2 was mainly different at R1 of C-3, while GA-C2 exhibited only mildly inhibitory effects on day 12 and had an inhibition ratio of $13.94 \%$. Furthermore, the difference between GA-B and GA-A was that the beta hydroxyl group was replaced at C-3 and the ketone group was replaced at C-15, and GA-B showed no in vitro activity at all. On the other hand, the R1 substituent at C-3 of GA-F showed limited similarity with that of GA-A, and GA-F also showed mild activity, which further suggested the importance of the keto group at C-3. We speculated that the keto group at C-3 and the alpha hydroxyl group at C-15 might be necessary for anti-cyst activity.

Group 2 compounds have double bonds at C-20 and keto groups at C-11 [30, 31]. The MDCK cyst screening test showed no anti-cyst activity for the four monomers in group 2, and they even showed a tendency to increase cyst growth. Although these compounds were included in the studies to uncover anti-tumor effects of GA [32], none of these four GA were effective in the MDCK cyst model. This result suggested the importance of the structure of the main core ring of Ganoderic acid; when C-11 was replaced by a keto group, it became inactive.
GA-DM, representing group 3, possesses double bonds between $\mathrm{C}-24$ and $\mathrm{C}-25$ and has a hydroxy group and no substituent at C-23 [33]. There are two double bonds at C-7(8) and C-9(11) and no double bonds between C-8 and C-9 in compound GA-T of group 4 [34]. GA-DM and GA-T showed significant inhibitory activity that was stronger than that of GA-F and GA-G. The main ring structure of GA-DM was the same as that in the first group, but GA-T possessed its own special structure. In terms of the structure-activity relationship of GA-T, the in vitro activity may be related to its specific characteristics and require deeper analysis. Compared with GA-A, only R1 and R4 among the GADM side chain groups are the same, and R6 is similar. These results indicate that when the main core group of GAs contain particular side chains, especially the keto group at $\mathrm{C}-3$ and the alpha hydroxyl group at C-15, ADPKD cyst development is inhibited.

During our initial screening, GA-A stood out among the 12 purified GA monomers because of its optimal in vitro activity in the MDCK cyst model. GA-A has been found to suppress the proliferation, metastasis and adhesion of breast cancer cells, prostate cancer cells, pancreatic cancer cells, etc., in previous studies [35-37]. The mechanisms by which GA-A inhibits cell proliferation and viability and accelerates cell apoptosis mainly involve a decrease in Cdk4, which targets the STAT3 and Wnt proteins [35-37].

As GT slows cyst development via the downregulation of the Ras/MAPK signaling pathway without affecting the mTOR signaling pathway [12], we evaluated the effect of GA-A on the Ras/ MAPK signaling pathway in cultured MDCK cells. The experimental 
results showed that FSK treatment upregulated Ras/B-raf/MEK/ERK and suppressed Raf-1 significantly [38]. The expression of downstream proliferation-associated genes, including Egr-1 and c-fos, was also increased. GA-A reversed all these intracellular signaling events. GA-A also downregulated the excessively activated Ras/ MAPK signaling pathway in the kidneys in the PKD mouse model.

The expression of PCNA is regarded as a marker of cellular proliferation and is usually upregulated in ADPKD [39]. Our results showed that GA-A also reduced the excessive activation of PCNA, suggesting that GA-A may play a role in inhibiting the abnormal proliferation of cyst epithelial cells in ADPKD.

The metabolism and pharmacokinetics of GA-A have been well studied, and GA-A is rapidly absorbed into the circulation ( $t_{\max }$ $0.25 \mathrm{~h})$ and eliminated relatively slowly $\left(t_{1 / 2}, 1.40 \mathrm{~h}\right)$ after intravenous administration in rats. The absolute oral bioavailability of GA-A was estimated to be $8.68 \%$ [40]. The limitation for its use was the poor oral bioavailability, which could be improved by the use of modern formulations and pharmaceutical techniques such as nanoparticles and crystal preparations. The metabolic kinetics of the main GA-A metabolites have been investigated, and GA-C2 is the most abundant reduction product of GA-A [41]. As we have discussed above, GA-C2 showed very low activity in vitro, which further emphasized the effect of GA-A itself on ADPKD. Furthermore, GA-A content accounts for more than half of $G$. lucidum, and the determination of GA-A content has been used as the scientific basis for judging the quality of G. lucidum; however, the specific content varies according to the differences in the origin and growth stage $[42,43]$. Hence, GA-A shows promise in terms of its market value and production potential.

In summary, our experimental data found that GA-A, as the most promising active monomer in GT, inhibited renal cyst development. GA-A attenuated renal cyst development by downregulating the intracellular Ras/MAPK signaling pathway and inhibiting cyst epithelial cell proliferation. GA-A shows great potential for development as a novel therapeutic agent for treating PKD.

\section{ACKNOWLEDGEMENTS}

This work was supported by the National Natural Science Foundation of China grants 81620108029, 81330074, 81261160507, 81170632, and 81873597 and the Beijing Natural Science Foundation grant 7172113. We thank Peter Igarashi and Stefan Somlo for the Ksp-Cre and $P k d 1^{\text {flox/flox }}$ mice.

\section{AUTHOR CONTRIBUTIONS}

$\mathrm{JM}$ and BXY designed the study; JM, JZH, SZ, BYH, SYW, SZW, SQL, ML and HZ performed the experiments and analyzed the data; JM and BXY wrote the paper.

\section{ADDITIONAL INFORMATION}

Competing interests: The authors declare no competing interests.

\section{REFERENCES}

1. Cornec-Le Gall E, Alam A, Perrone RD. Autosomal dominant polycystic kidney disease. Lancet. 2019;393:919-35.

2. Chapman AB, Devuyst O, Eckardt KU, Gansevoort RT, Harris T, Horie $S$, et al. Autosomal-dominant polycystic kidney disease (ADPKD): executive summary from a Kidney Disease: Improving Global Outcomes (KDIGO) Controversies Conference. Kidney Int. 2015;88:17-27.

3. Consortium T. I. P. K. D. Polycystic kidney disease: the complete structure of the PKD1 gene and its protein. Cell. 1995;81:289-98.

4. Mochizuki T, Wu G, Hayashi T, Xenophontos SL, Veldhuisen B, Saris JJ, et al. PKD2, a gene for polycystic kidney disease that encodes an integral membrane protein. Science. 1996;272:1339-42.

5. Harris PC, Torres VE. Polycystic kidney disease. Annu Rev Med. 2009;60:321-37.

6. Lee SH, Somlo S. Cyst growth, polycystins, and primary cilia in autosomal dominant polycystic kidney disease. Kidney Res Clin Pract. 2014;33:73-8.
7. Cornec-Le Gall E, Audrezet MP, Chen JM, Hourmant M, Morin MP, Perrichot R, et al. Type of PKD1 mutation influences renal outcome in ADPKD. J Am Soc Nephrol. 2013;24:1006-13.

8. Reule S, Sexton DJ, Solid CA, Chen SC, Collins AJ, Foley RN. ESRD from autosomal dominant polycystic kidney disease in the United States, 2001-2010. Am J Kidney Dis. 2014;64:592-9.

9. Ong AC, Devuyst O, Knebelmann B, Walz G. Autosomal dominant polycystic kidney disease: the changing face of clinical management. Lancet. 2015;385:1993-2002.

10. Edwards ME, Chebib FT, Irazabal MV, Ofstie TG, Bungum LA, Metzger AJ, et al. Long-term administration of tolvaptan in autosomal dominant polycystic kidney disease. Clin J Am Soc Nephrol. 2018;13:1153-61.

11. Lanktree MB, Chapman AB. New treatment paradigms for ADPKD: moving towards precision medicine. Nat Rev Nephrol. 2017;13:750-68.

12. Su L, Liu L, Jia Y, Lei L, Liu J, Zhu S, et al. Ganoderma triterpenes retard renal cyst development by downregulating Ras/MAPK signaling and promoting cell differentiation. Kidney Int. 2017;92:1404-18.

13. Rios JL, Andujar I, Recio MC, Giner RM. Lanostanoids from fungi: a group of potential anticancer compounds. J Nat Prod. 2012;75:2016-44.

14. Wu GS, Guo JJ, Bao JL, Li XW, Chen XP, Lu JJ, et al. Anti-cancer properties of triterpenoids isolated from Ganoderma lucidum - a review. Expert Opin Investig Drugs. 2013;22:981-92.

15. Gill BS, Navgeet, Kumar S. Ganoderic acid targeting multiple receptors in cancer: in silico and in vitro study. Tumour Biol. 2016;37:14271-90.

16. Gill BS, Navgeet, Mehra R, Kumar V, Kumar S. Ganoderic acid, lanostanoid triterpene: a key player in apoptosis. Investig New Drugs. 2018;36:136-43.

17. Xu JW, Zhao W, Zhong JJ. Biotechnological production and application of ganoderic acids. Appl Microbiol Biotechnol. 2010;87:457-66.

18. Yang HL. Ganoderic acid produced from submerged culture of Ganoderma lucidum induces cell cycle arrest and cytotoxicity in human hepatoma cell line BEL7402. Biotechnol Lett. 2005;27:835-8.

19. Torres VE, Wang X, Qian Q, Somlo S, Harris PC, Gattone VH 2nd. Effective treatment of an orthologous model of autosomal dominant polycystic kidney disease. Nat Med. 2004;10:363-4.

20. Wang W, Geng X, Lei L, Jia Y, Li Y, Zhou H, et al. Aquaporin-3 deficiency slows cyst enlargement in experimental mouse models of autosomal dominant polycystic kidney disease. FASEB J. 2019;33:6185-96.

21. Zhou H, Gao J, Zhou L, Li X, Li W, Li X, et al. Ginkgolide B inhibits renal cyst development in in vitro and in vivo cyst models. Am J Physiol Ren Physiol. 2012;302:F1234-42

22. Sun $Y$, Zhou H, Yang BX. Drug discovery for polycystic kidney disease. Acta Pharmacol Sin. 2011;32:805-16.

23. Wang W, Li F, Sun Y, Lei L, Zhou H, Lei T, et al. Aquaporin-1 retards renal cyst development in polycystic kidney disease by inhibition of Wnt signaling. FASEB J. 2015;29:1551-63.

24. Lei L, Wang W, Jia Y, Su L, Zhou H, Verkman AS, et al. Aquaporin-3 deletion in mice results in renal collecting duct abnormalities and worsens ischemiareperfusion injury. Biochim Biophys Acta, Mol Basis Dis. 2017;1863:1231-41.

25. Liang C, Tian D, Liu Y, Li H, Zhu J, Li M, et al. Review of the molecular mechanisms of Ganoderma lucidum triterpenoids: Ganoderic acids A, C2, D, F, DM, X and Y. Eur Jed Chem. 2019;174:130-41.

26. Kubota T, Asaka Y, Miura I, Mori H. Structures of ganoderic acid A and B, two new lanostane type bitter triterpenes from Ganoderma lucidum (FR.) KARST. Helv Chim Acta. 1982;65:611-9.

27. Kikuchi T, Kanomi S, Kadota S, Murai Y, Tsubono K, Ogita Zl. Constituents of the fungus Ganoderma lucidum (Fr.) Karst. I Structures of ganoderic acids C2, E, I, and K, lucidenic acid F and related compounds. Chem Pharm Bull. 1986;34:3695-712.

28. Kikuchi T, Matsuda S, Kadota S, Murai Y, Ogita Z. Ganoderic acid D, E, F, and H and lucidenic acid D, E, and F, new triterpenoids from Ganoderma lucidum. Chem Pharm Bull. 1985;33:2624-7.

29. Yue QX, Cao ZW, Guan SH, Liu XH, Tao L, Wu WY, et al. Proteomics characterization of the cytotoxicity mechanism of ganoderic acid $D$ and computerautomated estimation of the possible drug target network. Mol Cell Proteom. 2008;7:949-61

30. Che XQ, Li SP, Zhao J. Ganoderma triterpenoids from aqueous extract of Ganoderma lucidum. Zhongguo Zhong Yao Za Zhi. 2017;42:1908-15.

31. Li BM, Gu HF, Li Y, Liu C, Wang HQ, Kang J, et al. Determination of nine triterpenoid acids from Ganoderma lucidum of different producting areas by HPLC. Zhongguo Zhong Yao Za Zhi. 2012;37:3599-603.

32. Liu DL, Li YJ, Yang DH, Wang CR, Xu J, Yao N, et al. Ganoderma lucidum derived ganoderenic acid $B$ reverses $A B C B 1$-mediated multidrug resistance in HepG2/ ADM cells. Int J Oncol. 2015;46:2029-38.

33. Miyamoto I, Liu J, Shimizu K, Sato M, Kukita A, Kukita T, et al. Regulation of osteoclastogenesis by ganoderic acid DM isolated from Ganoderma lucidum. Eur J Pharmacol. 2009;602:1-7. 
34. Tang W, Liu JW, Zhao WM, Wei DZ, Zhong JJ. Ganoderic acid T from Ganoderma lucidum mycelia induces mitochondria mediated apoptosis in lung cancer cells. Life Sci. 2006;80:205-11.

35. Gill BS, Kumar S, Navgeet. Evaluating anti-oxidant potential of ganoderic acid A in STAT 3 pathway in prostate cancer. Mol Biol Rep. 2016;43:1411-22.

36. Yao $\mathrm{X}$, Li G, Xu H, Lu C. Inhibition of the JAK-STAT3 signaling pathway by ganoderic acid $A$ enhances chemosensitivity of HepG2 cells to cisplatin. Planta Med. 2012;78:1740-8.

37. Jiang J, Grieb B, Thyagarajan A, Sliva D. Ganoderic acids suppress growth and invasive behavior of breast cancer cells by modulating AP-1 and NF-kappaB signaling. Int J Mol Med. 2008;21:577-84.

38. Rushworth LK, Hindley AD, O'Neill E, Kolch W. Regulation and role of Raf-1/B-Raf heterodimerization. Mol Cell Biol. 2006;26:2262-72.
39. Elliott J, Zheleznova NN, Wilson PD. c-Src inactivation reduces renal epithelial cell-matrix adhesion, proliferation, and cyst formation. Am J Physiol Cell Physiol. 2011;301:C522-9.

40. Cao FR, Feng L, Ye LH, Wang LS, Xiao BX, Tao X, et al. Ganoderic acid A metabolites and their metabolic kinetics. Front Pharmacol. 2017:8:101.

41. Cao FR, Xiao BX, Wang LS, Tao X, Yan MZ, Pan RL, et al. Plasma and brain pharmacokinetics of ganoderic acid $A$ in rats determined by a developed UFLCMS/MS method. J Chromatogr B Anal Technol Biomed Life Sci. 2017;1052:19-26.

42. Lu J, Qin JZ, Chen P, Chen X, Zhang YZ, Zhao SJ. Quality difference study of six varieties of Ganoderma lucidum with different origins. Front Pharmacol. 2012;3:57. https://doi.org/10.3389/fphar.2012.00057.

43. Nakagawa T, Zhu Q, Tamrakar S, Amen Y, Mori Y, Suhara H, et al. Changes in content of triterpenoids and polysaccharides in Ganoderma lingzhi at different growth stages. J Nat Med. 2018;72:734-44. 